\title{
Reducing Breast Cancer Induction Risk from Chest CT Scanning Exams
}

\author{
Parinaz Mehnati ${ }^{1, *}$, Reza Malekzadeh ${ }^{2}$
}

${ }^{1}$ Department of Medical Physics, School of Medicine, Tabriz University of Medical Sciences, Tabriz, Iran

* Corresponding author: Parinaz Mehnati, Department of Medical Physics, School of Medicine, Tabriz University of Medical Sciences, Tabriz, Iran. E-mail:

DOI: $10.21859 /$ mci-supp-117 parinazmehnati@yahoo.com

\section{Keywords:}

Breast Cancer

Chest CT Exam

Bismuth Shielding

\begin{abstract}
Introduction: In Diagnostic assays of computed tomography of the lungs and heart, the sensitive breast tissue place in the main beam field, but most of the times, it is not as considered organ for diagnosis. Anatomically location of the breast in the imaging field in women, particularly in reproductive age, increases the risk of cancer induction due to ionizing radiation. Using shields are one of the solutions of radiation protection. Bismuth shielding is a practical method of radiation protection due to its special characteristics that can be while maintaining image diagnostic value, reduce radiation dose received by the breast tissue. In this study, we investigated the efficiency of bismuth shields in diagnostic methods and dose reduction amount to protect the breast in chest CT scan exams.
\end{abstract}

Materials and Methods: The published paper between 2006 and 2016 that were investigated on the bismuth shielding for breast dose reduction in computed tomography scanner types were enrolled. Bismuth from F\&L Company by using two types of anthropomorphic Phantom were used. TLD dosimeters and ionization chamber in the same exposure conditions used to assess the amount of breast dose reduction.

Results: Different types of CT scanners including 16, 64 and 256 slices showed a decline of breast tissue dose by the variation in the value. Average of 33, 49 and $38 \%$ dose reduction in breast tissue have been reported when using bismuth shielding in CT scans of 16, 64 and 256 slices, respectively.

Conclusions: The results indicated that the use of bismuth shielding in all CT scanners while maintaining image quality, giving a significant dose reduction in the breast. Radiation breast dose reduction can decline the risk of breast cancer induction by radiation possible be performed with this method. 